\title{
Vacuum-ultraviolet photoabsorption imaging system for laser plasma plume diagnostics
}

\author{
J. S. Hirsch, E. T. Kennedy, A. Neogi, and J. T. Costelloa) \\ National Centre for Plasma Science and Technology (NCPST) and School of Physical Sciences, Dublin City \\ University, Glasnevin, Dublin 9, Ireland \\ P. Nicolosi and L. Poletto \\ INFM - National Institute for Matter Physics, University of Padova, 35131 Padova, Italy
}

(Received 8 January 2003; accepted 20 February 2003)

\begin{abstract}
We describe a recently designed and constructed system based on a $1 \mathrm{~m}$ normal incidence vacuum monochromator with corrected (toroidal) optics that produces a wavelength tuneable and collimated vacuum-ultraviolet (VUV) $(\lambda=30-100 \mathrm{~nm})$ beam. The VUV continuum source is a laser-generated gold plasma. The primary function of the system is the measurement of time resolved "images" or spatial distributions of photoabsorption/photoionization in expanding laser plasma plumes. This is achieved by passing the beam through the sample of interest (in our case a second synchronised plasma) and recording the "footprint" of the attenuated beam on a charge coupled device. Using this VUV photoabsorption imaging or "shadowgraphy" technique we track and extract column density distributions in expanding plasma plumes. We can also measure the plume front velocity. We have characterized the system, particularly in relation to spectral and spatial resolution and the experimental results meet very well the expectations from ray tracing done at the design phase. We present first photoabsorption images and column density distributions of laser produced Ca plumes from the system. (C) 2003 American Institute of Physics. [DOI: 10.1063/1.1571974]
\end{abstract}

\section{INTRODUCTION}

The interaction of intense laser beams with materials, leading to rapid phase transitions and the production of hot plumes of material, is a subject almost as old as the laser itself. Although the plumes are intrinsically of interest as archetypal systems for the study of matter at high temperature, it has only been in the last decade or so that the potential of laser generated plasma plumes to find real application areas has been realized in, e.g., materials deposition, ${ }^{1}$ deepultraviolet (UV) to x-ray source in analytical spectroscopy, ${ }^{2}$ lithography, ${ }^{3}$ microscopy, ${ }^{4}$ etc. As a result experiments aimed toward "complete" spatio-temporal characterization of laser plasma plumes have become de rigeur in recent times. Direct imaging of the UV - near infrared light emitted from plasma plumes with gated charge coupled device (CCD) cameras ${ }^{5,6}$ and also of the light absorbed from wavelength tuned lasers ${ }^{7}$ are important diagnostics of laser ablated/plasma plumes. The former provides space and time resolved information on excited states of atomic and molecular species, while the latter can provide corresponding data on ground state or "dark" species. However, tabletop tuneable lasers are limited for many practical purposes to wavelengths in excess of 300 $\mathrm{nm}$ if one requires pulse energies above a few $\mathrm{mJ}$. As a result the range of accessible resonance lines of even singly charged ions is severely curtailed while reaching the ionization thresholds of these species is impossible.

A well established technique for the measurement of

\footnotetext{
a) Author to whom correspondence should be addressed; electronic mail: jtc@physics.dcu.ie
}

photoabsorption spectra of almost all atoms and ions is the dual laser plasma (DLP) method. ${ }^{8,9}$ In DLP spectroscopic experiments, one laser plasma constitutes the absorbing "sample" while the other forms the vacuum ultraviolet (VUV) (continuum) backlighting source. ${ }^{10}$ VUV light passed through the sample plasma is dispersed by a spectrometer and recorded on a VUV sensitive array detector. By varying the delay between the formation of the "sample" and "light source" plasmas one can measure the time resolved photoabsorption spectrum of the sample plasma. By recording photoabsorption spectra in different spatial zones of the sample plasma it is possible to build up a picture of how the plasma evolves in space and time. Unfortunately the use of the conventional DLP technique as a plasma diagnostic is very limited due to the necessity to probe sequentially the different plasma zones. The full spatial history of the plasma evolution must therefore be built up over many shots.

We have recently shown ${ }^{11}$ in a proof of principle experiment that probing a laser ablated/plasma plume with monochromatic VUV light can overcome this limitation. In contrast to the usual DLP spectroscopy experiment we pass a collimated VUV beam, tuned to an atomic or ionic resonance, through a laser-plasma plume and measure the resultant transmitted image (or shadow). In this way we are able to obtain directly the spatial-temporal distribution of particular plasma species.

As outlined in Ref. 11, VUV radiation has a number of attractive features for application to laser ablated/plasma plume characterization, some of which are worth repeating here for completeness: VUV light can access resonance lines of all atoms and moderately charged ions. Hence one can 
track plume species with resonance energies up to the photon energy limit of $35 \mathrm{eV}(\lambda \sim 40 \mathrm{~nm})$ here. The pulsed laser plasma light source emits VUV radiation typically for 0.1-50 ns, depending on the heating pulse duration; hence the temporal resolution available can be short and achieved automatically without any additional fast switching requirement. The laser plasma source makes analysis of the transmitted light distribution relatively uncomplicated. It is, to a very good approximation, a direct image of photoabsorption within the plume. There are essentially two reasons for this. The dimensions and effective relative bandwidth of a laser plasma source means that it is essentially an incoherent source (longitudinal coherence of $<10 \mu \mathrm{m}$ ) and hence image analysis is not complicated by the presence of interference patterns. In addition, refraction of a VUV beam in a plasma with a given density gradient is significantly reduced compared to the case for a visible beam, with beam deviation angles scaling approximately as $\left(\lambda_{\text {probe }}\right)^{2}$. This also means that one can access areas of the plasma in and around the critical density region of the plasma.

The use of VUV radiation as a probe has yet another very important advantage - it can be used to photoionize atoms or ions, a process effectively immune from the usual radiation transfer problems, which complicate optical probing of plasmas with ground state (bound) resonance transitions. In particular, tuneable VUV radiation can be used to induce resonant photoionization in, e.g., $\mathrm{Ba}^{+}$:

$$
\begin{aligned}
\mathrm{Ba}^{+} & \left(5 p^{6} 6 s^{2} S\right)+h \nu-\mathrm{Ba}^{+*}\left(5 p^{5} 6 s 5 d^{2} P\right) \\
& -\mathrm{Ba}^{2+}\left(5 p^{61} S\right)+e^{-} .
\end{aligned}
$$

Almost all absorbed photons are converted to electrons and hence one does not have to be concerned about multiple photon absorption/reemission cycles (opacity effects) in the plasma plume. This is a significant advantage of the VUV photoionization imaging technique. For those atoms (and ions) for which measured photoionization cross sections are available one can extract column density values (NL). (NL is the column density which is the integrated ion density times the absorbing column length along the line of sight of the VUV beam.) The use of inner shell excitations brings another advantage; excitation energies for different stages of ionization tend to be displaced slightly from each other. Thus, keeping all other experimental conditions identical, one can readily image different ionization stages of the same atom in one experiment by tuning the monochromator energy to the relevant resonance lines.

In Ref. 11, we employed a diverging beam interrogated by a back-thinned CCD array. A more ideal configuration would use a collimated beam. The advantage of probing the sample plasma with a parallel beam is that it simplifies the geometrical interpretation of photoabsorption images and thus the extraction of physical information. Moreover it gives us the freedom to place the sample plasma and/or detector (a backthinned CCD), at variable distances from the collimating mirror chamber. This is not a trivial point. Apart from flexibility in attaching "end stations" to the beam, in those wavelength regions where the intensity of the continuum source is low, the signal due to the front plasma

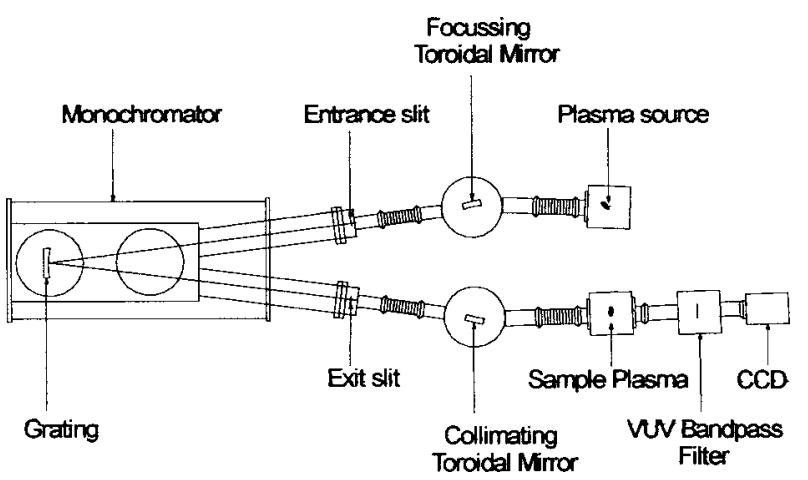

FIG. 1. Optical layout of the vacuum ultraviolet photoabsorption imaging system.

emission can be of comparable intensity. By placing the detector at a large distance from the sample plasma, the unwanted emission from it falling on the CCD detector can be significantly reduced (compared to the VUV probe beam intensity), thereby improving the signal to noise ratio of the photoabsotpiom images and thus the accuracy of the measurements.

We report here a tuneable and collimated VUV beam system based on a $1 \mathrm{~m}$ normal incidence vacuum monochromator coupled to a laser plasma VUV continuum source. A specially designed toroidal mirror couples radiation from a laser plasma light source onto the entrance slit of the monochromator while a second mirror collimates the wavelength tuned (VUV) radiation emerging from the exit slit. The latter monochromatic VUV beam is used to probe the sample of interest located downstream between the collimating mirror and $\mathrm{CCD}$ detector.

We first present the basic platform and describe the main considerations determining the final design. Measurements of the key parameters for the system, namely spectral resolution, spatial resolution, and VUV flux are presented and compared to the original values predicted by our ray tracing design code. Finally, to illustrate the usefulness of the system, we show some time resolved images of $\mathrm{Ca}$ and $\mathrm{Ba}$ plasmas and a column density map for $\mathrm{Ba}^{+}$is derived.

\section{BASIC OPTICAL PLATFORM}

A schematic diagram of the system is shown in Fig. 1. The main elements are the VUV light continuum light source (W or Au plasma), gold coated focusing and collimating toroids, normal incidence monochromator (Acton Research ${ }^{\mathrm{TM}}$ model VM-521), sample (Ca and Ba here), and back illuminated CCD (Andor-Technology ${ }^{\circledR}$ ). ${ }^{12}$ All five major component parts are housed in vacuum chambers and evacuated with Leybold PT-series ${ }^{\mathrm{TM}}$ turbomolecular pumping rigs to pressures of better than $1 \times 10^{-5}$ mbar. The VUV continuum emitting plasma is formed by focusing the output pulse (800 $\mathrm{mJ} / 1064 \mathrm{~nm} / 8 \mathrm{~ns}$ ) from a Continuum Surelite ${ }^{\mathrm{TM}} \mathrm{Nd}-\mathrm{yttrium}-$ aluminum-garnet (YAG) laser onto tungsten or gold slabs. The spot diameter is $<100 \mu \mathrm{m}$ and hence the irradiance approaches $1 \mathrm{TW} \mathrm{cm}{ }^{-2}$. The VUV pulse duration is $\sim 50 \mathrm{~ns}$ and determines the system time resolution. For better time resolution we plan to use another Nd-YAG laser (EKSPLA $312 \mathrm{P}^{\mathrm{TM}}$ ) which delivers up to $400 \mathrm{~mJ}$ per laser shot in $170 \mathrm{ps}$ 
at $1064 \mathrm{~nm}$. Based on earlier measurements on continuum generation with plasmas formed by picosecond lasers ${ }^{13}$ we expect this laser plasma driver to yield a sub 250 ps VUV pulse. The absorbing (sample) plasma is formed by focusing the $300 \mathrm{~mJ} / 1064 \mathrm{~nm} / 15 \mathrm{~ns}$ output pulse from a Spectron SL400 ${ }^{\mathrm{TM}}$ laser to a line of length $3 \mathrm{~mm}$ and height $0.1 \mathrm{~mm}$ onto Ca slabs. The resultant peak on-target irradiance is $\sim 6$ $\mathrm{GW} \mathrm{cm}^{-2}$.

A major drawback of the earlier system ${ }^{11}$ was the low intensity of the VUV beam. The main reason was the low reflectivity in the 30-100 nm wavelength range of the focusing spherical mirror used and also the aberrations introduced by having to operate it off-normal incidence. Coupled with the relatively low intensity of the continuum source of $10^{14}$ photons $/ \mathrm{nm} / \mathrm{Sr},{ }^{14}$ obtaining images with good signal to noise ratio, was difficult. As a consequence, to counterbalance the lack of light flux, both entrance and exit slits of the $0.2 \mathrm{~m}$ monochromator had to be opened to $250 \mu \mathrm{m}$ resulting in poor spectral resolution ( $2 \mathrm{~nm}$ at a wavelength of $50 \mathrm{~nm}$ ). In the VUV $\rightarrow$ soft $x$-ray spectral range, optical systems operating at grazing incidence are widely used to enhance reflectivity. For these angles of incidence, astigmatism is very important and without careful design a loss in spectral and/or spatial resolution can result. In systems using concave spherical gratings, a matched toroidal mirror, placed between the light source and grating, can be used to eliminate or at least significantly reduce astigmatism. ${ }^{15}$ A new optical layout comprising two gold-coated toroidal mirrors and a VUV monochromator, for which parameters have been defined using a ray tracing code, ${ }^{16}$ is used here to produce a system with relatively high spectral and spatial resolution while maintaining optimum VUV flux throughput.

The wavelength of interest is selected using a $1 \mathrm{~m}(R$ $=999.94 \mathrm{~mm}$ ) normal incidence monochromator with a concave grating of 1200 groves $/ \mathrm{mm}$. The grating is blazed for a wavelength of $80 \mathrm{~nm}$ and covers the wavelength range between 30 and $150 \mathrm{~nm}$. The reciprocal linear dispersion is 0.8 $\mathrm{nm} / \mathrm{mm}$ at a wavelength of $50 \mathrm{~nm}$ and the best resolution attainable is $\sim 10000$ at $100 \mathrm{~nm}$ with $5 \mu \mathrm{m}$ wide entrance/ exit slits. The dimensions of the grating are $96 \mathrm{~mm} \times 56 \mathrm{~mm}$ and hence the aperture of the spectrometer is $f / 10.4$ in the tangential plane and $f / 17.8$ in the sagittal plane. The spectrometer is evacuated with a turbomolecular pumping system to a pressure of $\sim 1 \times 10^{-6}$ mbar.

Guided by an extensive ray tracing study, the parameters of the first toroidal mirror were chosen so as to focus the laser plasma light onto the entrance slit of the monochromator with minimal aberration. Space and mechanical constraints determined that the length of the entrance arm should be set at $400 \mathrm{~mm}$. This distance was also the minimum permitted to ensure that the mirror was not damaged by plasma debris.

The angle of incidence on the focussing mirror was set at $85^{\circ}$ where the reflectivity of gold is high $(78 \%$ at $50 \mathrm{~nm})$. The mirror operates in the Rowland circle configuration, i.e., the mirror to source distance is equal to the mirror to image distance in order to minimize aberrations. The aperture of the system was reduced to $10 \mathrm{mrad} \times 10 \mathrm{mrad}$ by masking the toroidal mirror on the entrance arm of the system, which is (a)

(b)

(c)

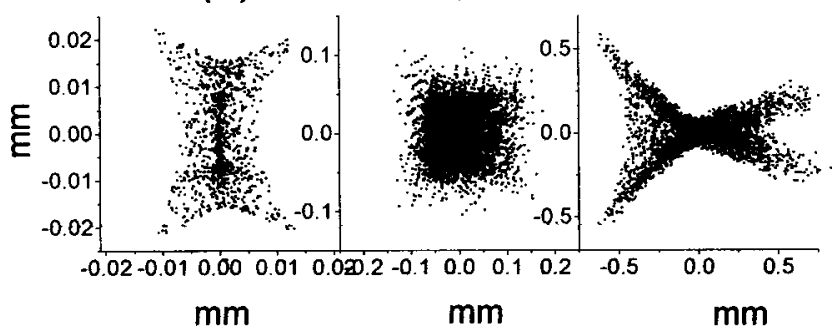

FIG. 2. Computer simulated vacuum ultraviolet "footprints" of the laser plasma source on a plane located at the entrance slit of the $1 \mathrm{~m}$ normal incidence monochromator for: (a) $85^{\circ}$ incidence and an aperture of $f / 100$ $\times f / 100$; (b) $85^{\circ}$ incidence and an aperture of $f / 50 \times f / 50$; and (c) $85^{\circ}$ incidence and an aperture of $f / 25 \times f / 25$. By aperturing down the focusing mirror on the entrance arm to $f / 100$ in both the sagittal and meridional directions, the footprint is less than $50 \mu \mathrm{m}$ on each side.

optimized to operate with $50 \mu \mathrm{m}$ wide entrance and exit slits in order to improve spectral resolution over that obtained in Ref. 11. Optical aberrations for point-like sources at the entrance slit plane of the monochromator have been simulated using the ray tracing code and are shown in Fig. 2. It is clear that at $85^{\circ}$ incidence, the aberrations are larger than $100 \mu \mathrm{m}$ for an angle of acceptance of $20 \mathrm{mrad} \times 20 \mathrm{mrad}$ and more than $1 \mathrm{~mm}$ for an acceptance angle of $40 \mathrm{mrad} \times 40 \mathrm{mrad}$. For an aperture of $10 \mathrm{mrad} \times 10 \mathrm{mrad}$, the size of the image of a point source is less than $40 \mu \mathrm{m}$, indicating little or no light flux loss at the $(50 \mu \mathrm{m})$ entrance slit of the monochromator for a small point-like plasma source.

The various mirror parameters are obtained by solving the equations ${ }^{17}$

$$
\frac{1}{p}+\frac{1}{q}=\frac{2}{R \cos \alpha}
$$

in the meridional plane

$$
\frac{1}{p}+\frac{1}{q^{\prime}}=\frac{2 \cos \alpha}{\rho},
$$

in the sagittal plane where $p$ is the mirror to source distance, $q$ the mirror to image distance in the meridional plane, $q^{\prime}$ the image to mirror distance in the sagittal plane, $\alpha$ the angle of incidence, while $R$ and $\rho$ are the tangential and sagittal radii of curvature, respectively. Having fixed $p, q$, and $q^{\prime}$, the other parameters of the toroidal mirror were calculated and summarized in Table I, the second column.

The parameters of the second toroidal mirror have been chosen to create a parallel beam of cross section $4 \mathrm{~mm} \times 4$

TABLE I. Entrance (focusing) and exit (collimating) arm toroidal mirror parameters.

\begin{tabular}{lcc}
\hline \hline Parameter & Focusing toroid & Collimating toroid \\
\hline Entrance arm & $400 \mathrm{~mm}$ & $400 \mathrm{~mm}$ \\
Exit arm & $400 \mathrm{~mm}$ & - \\
Tangential radius & $4590 \mathrm{~mm}$ & $9180 \mathrm{~mm}$ \\
Sagittal radius & $34.9 \mathrm{~mm}$ & $63.5 \mathrm{~mm}$ \\
Incidence angle & $85^{\circ}$ & $85^{\circ}$ \\
Coating & Gold & Gold \\
Mirror size & $60 \times 20 \mathrm{~mm}$ & $60 \times 20 \mathrm{~mm}$ \\
Angle of acceptance & $10 \times 10 \mathrm{mrad}$ & $10 \times 10 \mathrm{mrad}$ \\
\hline \hline
\end{tabular}


$\mathrm{mm}$. This condition, as well as the reduced numerical aperture of the system, determined the position of the mirror to be $400 \mathrm{~mm}$ from the exit slit of the monochromator. As for the focusing mirror on the input arm, using the ray tracing code to monitor the optical aberrations for different angles of incidence, the angle was set at $85^{\circ}$ where the aberrations were found to be negligible and the reflectivity of the gold coated mirror was high (typically 75\%-85\%). The parameters of the collimating mirror calculated using Eqs. (1) and (2) and are given in Table I, column 3. Both mirrors, placed in precision optical mounts allowing rotation and translation movements, are enclosed in cylindrical (aluminum) chambers and evacuated using a turbomolecular pump to pressure below $10^{-5}$ mbar. All the different components of the system are connected using ISO-KF 40 aluminum pipes and are all evacuated using turbomolecular pumps. Finally the image data acquisition is performed using a VUV sensitive, 2048 $\times 512$ pixel backthinned camera with a pixel size of 13 $\mu \mathrm{m} \times 13 \mu \mathrm{m}$.

\section{SYSTEM CHARACTERIZATION}

\section{A. Beam footprint}

The system was designed to deliver a collimated VUV beam of cross section $4 \mathrm{~mm} \times 4 \mathrm{~mm}$. Figures 3(a) and 3(b) show the calculated and measured footprint of the collimated VUV beam for different wavelengths $(30,50$, and $100 \mathrm{~nm})$ at distances of 550 and $1050 \mathrm{~mm}$ from the exit mirror. We observe a good agreement between the measured and calculated footprints. In particular the probe beam displays show a very low beam divergence $(\leqslant 0.5 \mathrm{mrad})$ in both the horizontal and vertical directions.

\section{B. Spectral resolution}

The spectral resolution of the system was calculated using the ray tracing program for different entrance and exit slit widths at wavelengths of $30,50,70$, and $100 \mathrm{~nm}$. The wavelength was scanned (numerically) in $0.01 \mathrm{~nm}$ steps in order to simulate the full width at half maximum of the transmitted intensity (computed instrument function). The results are shown in Fig. 4(a). We note that the resolving power is mainly determined by the width of the entrance slit of the monochromator when using the 1200 grooves $/ \mathrm{mm}$ grating. A best resolution of up to 10000 for a $5 \mu \mathrm{m}$ entrance slit is quoted by the manufacturer. With a $50 \mu \mathrm{m}$ entrance slit width, the resolving power is $\sim 1000$ at a wavelength of 50 $\mathrm{nm}$, and more than 2000 at $100 \mathrm{~nm}$. Based on these simulations we decided to operate the system with a $50 \mu \mathrm{m}$ entrance in order to capture almost all source flux. We set the exit slit (which determines both spectral and spatial resolution) at the same value having the option to increase the exit slit width to $100 \mu \mathrm{m}$ to gain more flux.

These computed results were checked experimentally using absorption spectroscopy. We measured on the He $1 s^{2}-1 s 2 p$ absorption resonance at $58.43 \mathrm{~nm}$. The helium pressure $(P)$ in the target chamber was held in the range 0.17 mbar $<P<0.19$ mbar and the absorbing column length (L) was $\sim 900 \mathrm{~mm}$. The experimental procedure is as follows; the pixels on the CCD camera were binned " $64 \times 64$ "

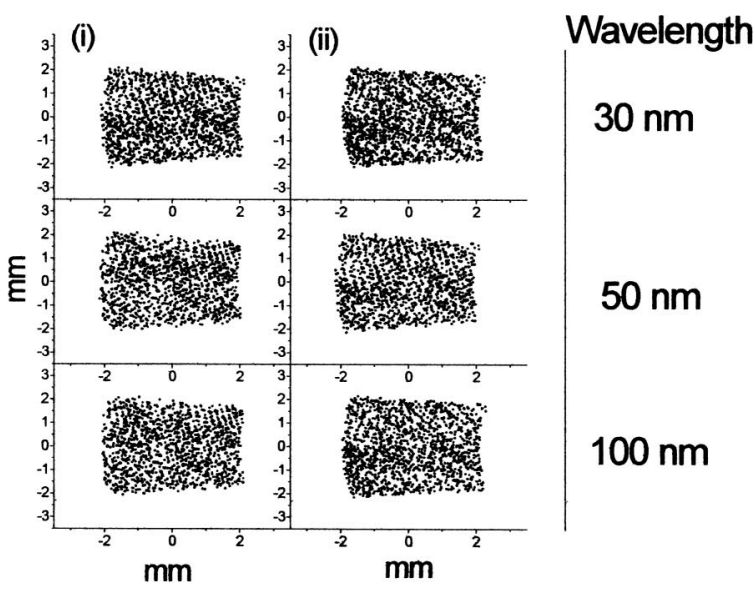

(a) (i)

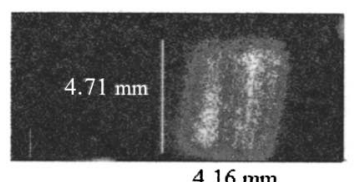

4. $16 \mathrm{~mm}$

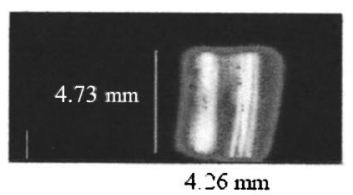

4. $26 \mathrm{~mm}$

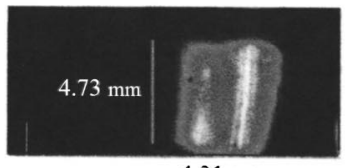

4. $21 \mathrm{~mm}$ (ii)
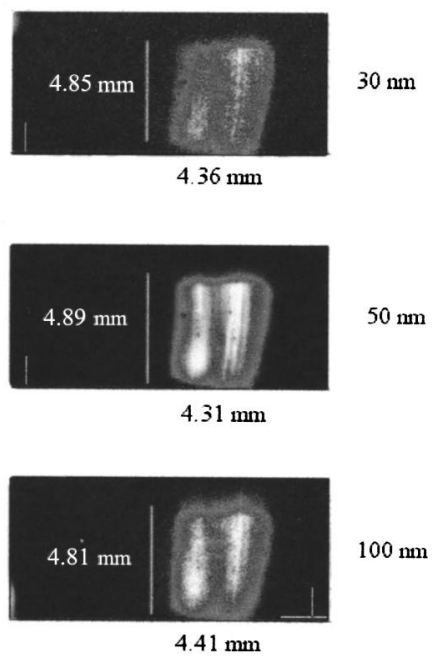

(b)
FIG. 3. Comparison between predicted (a) top panel and measured (b) bottom panel footprints of the collimated VUV beam for three different wavelengths $(30,50$, and $100 \mathrm{~nm})$ at two distances: (i) $550 \mathrm{~mm}$ and (ii) $1050 \mathrm{~mm}$ from the collimating mirror.

resulting in "superpixels" of size $0.832 \mathrm{~mm} \times 0.832 \mathrm{~mm}$ to maximize the detected flux. Due to the large area of the superpixels, single shot data acquisition was possible, thus preventing the formation of large craters on the target surface and giving us better stability on the output flux. A set of images were taken scanning the wavelength from 58.2 to $58.50 \mathrm{~nm}$ in $0.02 \mathrm{~nm}$ steps. Four $(64 \times 64)$ superpixels were isolated on every image and the total of count per superpixel added for every wavelength. The results are plotted in Fig. 4(b) for matched entrance and exit slits widths of 50 and 100 $\mu \mathrm{m}$ each. For entrance and exit slits of $100 \mu \mathrm{m}$, we measured a linewidth of $0.08 \pm 0.01 \mathrm{~nm}$ (full width at half maximum) while a linewidth of $0.05 \pm 0.01 \mathrm{~nm}$ FWHM was obtained when operating with entrance and exit slits of $50 \mu \mathrm{m}$ width. These experimental values correspond to resolving powers of 730 and 1160 at $58.43 \mathrm{~nm}$ and are in good agreement with ray tracing, which predicts resolving powers of 660 and 1200 , respectively, at $58.43 \mathrm{~nm}$. 


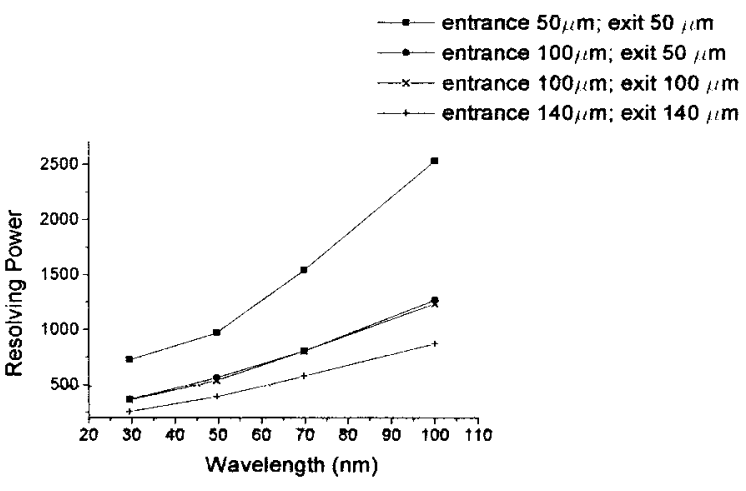

(a)
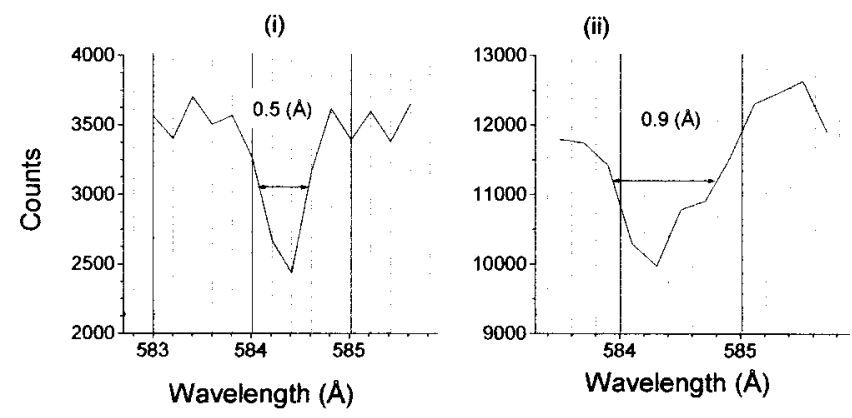

(b)

FIG. 4. Resolving power of the system predicted by ray tracing (a) upper panel. Results are shown for different combinations of entrance and exit slits for a number of wavelengths in the $30-100 \mathrm{~nm}$ range. Measured (absorption) line profiles (b) lower panel at the $58.4 \mathrm{~nm}\left(\mathrm{He} 1 s^{2}-1 s 2 p\right)$ resonance for $50 \mu \mathrm{m} / 50 \mu \mathrm{m}$ and $100 \mu \mathrm{m} / 100 \mu \mathrm{m}$ wide entrance/exit slits. The corresponding resolving powers of 1200 and 650 are in excellent agreement with the predicted values.

\section{Spatial resolution}

The horizontal (tangential plane) spatial resolution was measured using the edge trace technique. ${ }^{18}$ The edge trace was obtained by inserting a knife edge into the path of the collimated VUV beam for different combinations of entrance and exit slit widths. The knife edge is oriented parallel to slit jaws and can be translated horizontally on a micrometer driven stage. The edge trace is the integral of the line spread function $^{18}$ and hence the latter can be obtained by simply differentiating the edge trace. Figure 5(a) shows an image of the edge trace at a wavelength of $50 \mathrm{~nm}$ with entrance and exit slit widths of $100 \mu \mathrm{m}$. The figure is divided into two parts. On the left-hand side, the actual shadow of the VUV beam, showing the knife edge cutoff, recorded by the CCD camera is shown. The monochromator was set at a wavelength of $50 \mathrm{~nm}$ for these measurements. The pixels were binned $4 \times 4$ (effectively $52 \mu \mathrm{m} \times 52 \mu \mathrm{m}$ superpixels) and the image was recorded by accumulating ten laser shots. On the right-hand side a plot of intensity versus horizontal pixel number at a particular vertical pixel position is shown. The two horizontal lines on the plot are set at $10 \%$ and $90 \%$ of the total intensity and the slope of the intensity falloff is measured between these lines. With both entrance and exit slits set at $100 \mu \mathrm{m}$, the position of the intersection of the knife edge with the $10 \%$ and $90 \%$ lines is separated by $20 \pm 1$ pixels or $260 \pm 13 \mu \mathrm{m}$. In Fig. 5(b) we show a Lorentzian profile fitted to the corresponding line spread function ob-

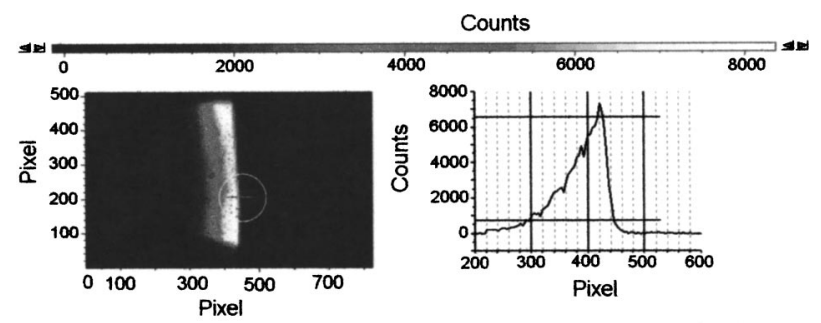

(a)

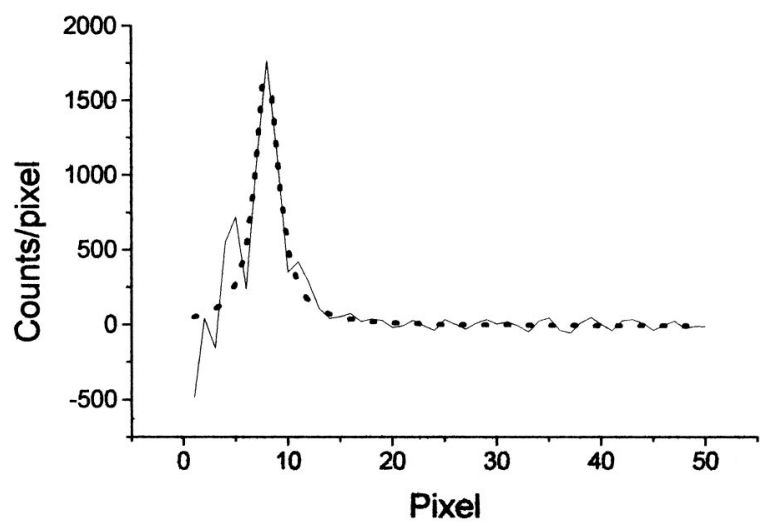

(b)

FIG. 5. Knife edge image (a) left-hand side panel and corresponding lineout (a) right-hand side panel measured at a wavelength of $50 \mathrm{~nm}$. The upper panel was obtained at $100 \mu \mathrm{m} / 100 \mu \mathrm{m}$ wide entrance/exit slits while the lower panel (b) shows a Lorentzian fit to the corresponding line spread function.

tained by differentiating this trace. This fit gives a FWHM of $135 \pm 30 \mu \mathrm{m}$, which is to be compared with a value of 130 $\mu \mathrm{m}$ from ray tracing with the same entrance/exit slit widths. The horizontal spatial resolution improves independently of the entrance slit width when the width of the exit slit is further reduced. An accurate measurement is, however, difficult to make since the flux at the CCD decreases accordingly. That said, ray tracing predicts that a tangential spatial resolution of $65 \mu \mathrm{m}$ should be possible for a point-like source and exit slit width of $50 \mu \mathrm{m}$ with the current system. The vertical spatial resolution (sagittal plane) was measured using the same procedure but with a horizontal knife edge. Here the resolution is limited by the emitting size of the plasma source. We measure a vertical resolution in the range $160 \pm 30 \mu \mathrm{m}$ which can be compared with a ray tracing prediction of $180 \mu \mathrm{m}$.

\section{VACUUM-UV PHOTOABSORPTION (PHOTOIONIZATION) IMAGES OF Ca PLASMA PLUMES}

\section{A. Time resolved absorption images of $\mathrm{Ca}, \mathrm{Ca}^{+}$, and $\mathrm{Ca}^{2+}$}

In Figs. 6(a), 6(b), and 6(c) we show a series of photoabsorption images of $\mathrm{Ca}$ plumes recorded at different instants after the plasma breakdown. The VUV beam was tuned to the inner shell $(3 p-3 d)$ resonances of atomic $\mathrm{Ca}$ [Fig. 6(a)] and $\mathrm{Ca}^{+}[$Fig. 6(b)] and also the valence $(3 p-3 d)$ resonance of $\mathrm{Ca}^{2+}$ [Fig. 6(c)] in order to separate out the different charges states. The VUV continuum duration is $\sim 30 \mathrm{~ns}$ and represents the exposure time for each recording. The shad- 

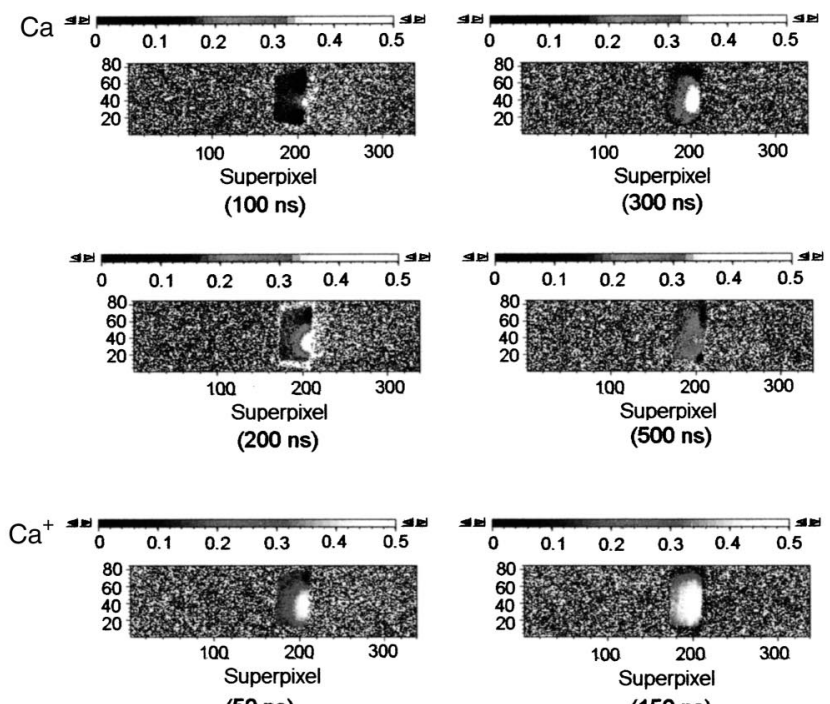

(50 ns)
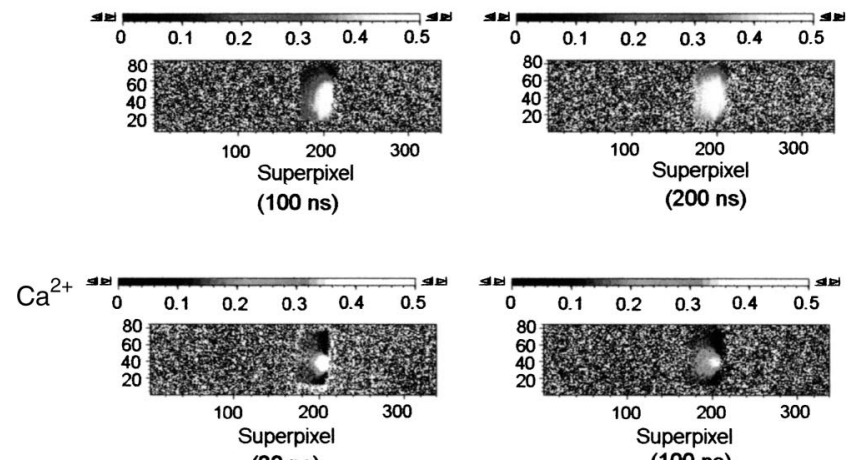

(20 ns)
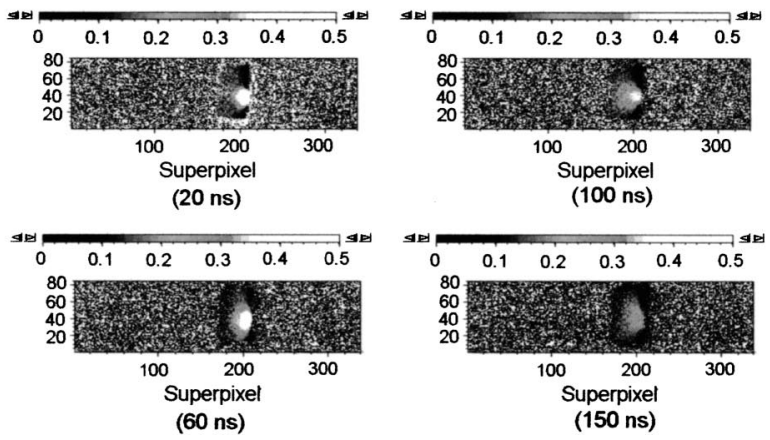

FIG. 6. Time resolved equivalent width distributions $\left(W_{E}\right)$ obtained from absorbance images $\left[\log _{10}(I o / I)\right]$ for atomic $\mathrm{Ca}, \mathrm{Ca}^{+}$, and $\mathrm{Ca}^{2+}$.

owgraphs embody information on the spatio-temporal evolution of the ground state species in the plume, i.e., the dark material not seen in time resolved emission imaging with a gated CCD camera. All pictures are photoexcitation in nature. However, since the $3 p-3 d$ resonances in the neutral and singly charged species are inner shell transitions, these images are effectively (resonant) photoionization images of these species. For the doubly charged ion, with an Ar-like electronic configuration of $3 p^{6}\left({ }^{1} S_{0}\right)$, the image is due to photoexcitation only and the excited state can decay only by fluorescence. The expansion of the individually selected (ground state) species within the Ca plume are clearly quite different. The $\mathrm{Ca}^{+}$component moves at a velocity of $\sim 2$ $\times 10^{6} \mathrm{~cm} \mathrm{~s}^{-1}$, while the atomic $\mathrm{Ca}$ component is clearly a good deal slower at $\sim 4 \times 10^{5} \mathrm{~cm} \mathrm{~s}^{-1}$.

\section{B. Measurement of plasma plume ion column densities- $\mathrm{Ba}^{+}$}

Where the total (absolute) photoionization cross section of an atom of ion has been measured we are also able to
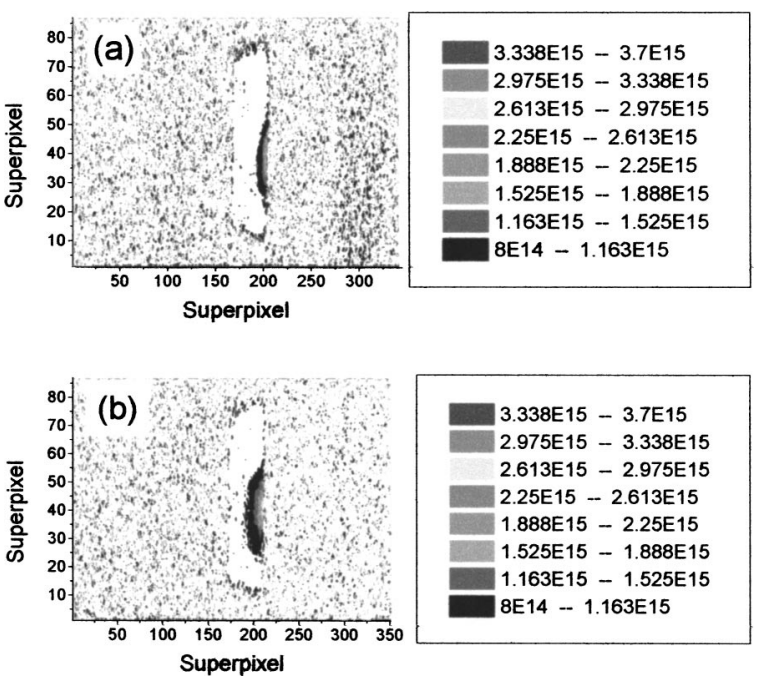

FIG. 7. Time resolved column density $\left[\mathrm{NL}=\int n(1) d l\right]$ maps of $\mathrm{Ba}^{+}$in a plasma plume at: (a) $100 \mathrm{~ns}$ and (b) $200 \mathrm{~ns}$ after the initiation of the plasma.

extract column densities, $\mathrm{NL}=\int n(x) d x$, where $n(x)$ is the ion density along the line of sight. Such data are available for $\mathrm{Ba}^{+19}$ while the procedure to obtain them has already been described in some detail ${ }^{11}$ and so we only give an outline here. Briefly we constructed "equivalent width" maps $W_{E}(x, y)$ from each shadowgram. Since the continuum intensity does not vary much over the spectral width of the probe beam (typically $\sim 0.1 \mathrm{~nm}$ ) the expression for the equivalent width simplifies to

$$
W_{E}=\int\{1-\exp [\sigma(E) \mathrm{NL}]\} d E,
$$

where $\sigma(E)$ is the total absolute photoionization cross section and the integral is taken over the $26.5 \mathrm{eV}$, tentatively assigned by us as the $5 p-6 d$ resonance profile. To convert the maps of equivalent width obtained experimentally into maps of column density we solved graphically the above equation. The profile of the absolute cross section is known ${ }^{19}$ and by multiplying $\sigma(E)$ by different values of NL we can compute the value of $W_{E}$ using a suitable software package (here MATLAB ${ }^{\text {TM }}{ }^{20}$ or MICROCAL ORIGIN ${ }^{\mathrm{TM}}{ }^{21}$ ). We constructed in this way a table of values of $W_{E}$ as a function of the column density. We then plotted NL versus $W_{E}$ and by fitting a high order polynomial to the curve we converted measured $W_{E}$ image (pixel) values directly into column density values. In Fig. 7 we show such "column density maps" for $\mathrm{Ba}^{+}$at 100-200 ns after plasma breakdown. The column densities are in the range $(1-3) \times 10^{15}$ ions $\mathrm{cm}^{-2}$ in the regions probed which lie between $\sim 0.5$ and $4 \mathrm{~mm}$ from the target surface. We will report in detail on these and other data obtained on $\mathrm{Ca}$ and $\mathrm{Ba}$ plasma plumes in a subsequent article.

\section{ACKNOWLEDGMENTS}

Work supported by the Higher Education Authority under the Program for Research in Third Level Institutions (PRTLI) and Enterprise Ireland under the Basic Research 
Grants Scheme. The authors have profited from useful discussions with Professor Ciaran Lewis of the Queen's University of Belfast, Northern Ireland.

${ }^{1}$ K. W. Mah, J.-P. Mosnier, E. McGlynn, M. O. Henry, D. O'Mahony, and J. G. Lunney, Appl. Phys. Lett. 80, 3301 (2002).

${ }^{2}$ M. A. Khater, P. van Kampen, J. T. Costello, J.-P. Mosnier, and E. T. Kennedy, J. Phys. D 33, 2252 (2000)

${ }^{3}$ H. M. Hertz, M. Berglund, B. A. M. Hansson, O. Hemberg, and G. J. Johansson, J. Phys. IV 11, 389 (2001).

${ }^{4}$ A. G. Michette, R. Fedosejevs, S. J. Pfauntsch, and R. Bobkowski, Meas. Sci. Technol. 5, 555 (1994).

${ }^{5}$ A. Neogi and R. K. Thareja, J. Appl. Phys. 85, 1131 (1999).

${ }^{6}$ D. B. Geohegan, A. A. Puretzky, G. Duscher, and S. J. Pennycook, Appl. Phys. Lett. 72, 2987 (1998).

${ }^{7}$ T. P. Williamson, G. W. Martin, A. H. El-Astal, A. Al-Khateeb, I. Weaver, D. Riley, M. J. Lamb, T. Morrow, and C. L. S. Lewis, Appl. Phys. A: Mater. Sci. Process. 69, S859 (1999).

${ }^{8}$ J. T. Costello, E. T. Kennedy, J.-P. Mosnier, P. K. Carroll, and G. O’Sullivan, Phys. Scr., T 34, 77 (1991).
${ }^{9}$ E. Jannitti, P. Nicolosi, and G. Tondello, Phys. Scr. 41, 458 (1990).

${ }^{10}$ P. K. Carroll, E. T. Kennedy, and G. O’Sullivan, Opt. Lett. 2, 72 (1978).

${ }^{11}$ J. S. Hirsch et al., J. Appl. Phys. 88, 4953 (2000).

${ }^{12}$ Details on Andor Technology CCD cameras are available at http:// www.andor-tech.com/main3.htm

${ }^{13}$ O. Meighan et al., J. Phys. B 33, 1159 (2000).

${ }^{14}$ J. Fischer, M. Kühne, and B. Wende, Appl. Opt. 23, 4252 (1984).

${ }^{15}$ P. Villoresi, P. Nicolosi, and M. Pelizzo, Appl. Opt. 39, 85 (2000).

${ }^{16}$ G. Bonfante, M.Sc. thesis, Universita degli Studi di Padova, 1989 (unpublished—details from LP at poletto@dei.unipd.it)

${ }^{17}$ J. A. R Samson, Vacuum Ultraviolet Spectroscopy (Cruithne, Glasgow, 2000).

${ }^{18}$ C. S. Williams and O. A. Becklund, Introduction to the Optical Transfer Function (Wiley Interscience, New York, 1989).

${ }^{19}$ I. C. Lyon, B. Peart, J. B. West, and K. Dolder, J. Phys. B 19, 4137 (1986).

${ }^{20}$ Information on Microcal Origin is available at http://www.originlab.com/ www/products/origin/index.asp

${ }^{21}$ Information on Matlab is available at http://www.mathworks.com/ programs/release13/index.shtml 